\title{
Middle-energy electron anisotropies in the auroral region
}

\author{
P. Janhunen ${ }^{1}$, A. Olsson ${ }^{2}$, H. Laakso ${ }^{3}$, and A. Vaivads ${ }^{2}$ \\ ${ }^{1}$ Finnish Meteorological Institute, Geophysical Research, Helsinki, Finland \\ ${ }^{2}$ Swedish Institute of Space Physics, Uppsala Division, Uppsala, Sweden \\ ${ }^{3}$ ESTEC, Space Science Department, Noordwijk, The Netherlands
}

Received: 22 January 2003 - Revised: 25 March 2003 - Accepted: 13 May 2003 - Published: 1 January 2004

\begin{abstract}
Field-aligned anisotropic electron distribution functions of $T_{\|}>T_{\perp}$ type are observed on auroral field lines at both low and high altitudes. We show that typically the anisotropy is limited to a certain range of energies, often below $1 \mathrm{keV}$, although sometimes extending to slightly higher energies as well. Almost always there is simultaneously an isotropic electron distribution at higher energies. Often the anisotropies are up/down symmetrical, although cases with net upward or downward electron flow also occur. For a statistical analysis of the anisotropies we divide the energy range into low (below $100 \mathrm{eV})$, middle $(100 \mathrm{eV}-1 \mathrm{keV})$ and high (above $1 \mathrm{keV}$ ) energies and develop a measure of anisotropy expressed in density units. The statistical magnetic local time and invariant latitude distribution of the middleenergy anisotropies obeys that of the average auroral oval, whereas the distributions of the low and high energy anisotropies are more irregular. This suggests that it is specifically the middle-energy anisotropies that have something to do with auroral processes. The anisotropy magnitude decreases monotonically with altitude, as one would expect, because electrons have high mobility along the magnetic field and thus, the anisotropy properties spread rapidly to different altitudes.
\end{abstract}

Key words. Magnetospheric physics (auroral phenomena). Space plasma physics (wave-particle interactions; changed particle motion and acceleration)

\section{Introduction}

Field-aligned electron anisotropies have been found on auroral field lines at both high and low altitudes. Below $8000 \mathrm{~km}$ altitude, field-aligned electrons were detected by the S3-3 satellite (Sharp et al., 1980; Collin et al., 1982). Close to the equatorial plane, bidirectional anisotropies were studied using IMP 6 (Hada et al., 1981), AMPTE/CCE (Klumpar et al., 1988), AMPTE/IRM (Sergeev et al., 2001) and SCATHA

Correspondence to: A. Olssen (ao@irfu.se)
(Richardson et al., 1981). Also geostationary satellites (corresponding to invariant latitude of $67^{\circ}$ ) have detected electron anisotropies (Lin et al., 1979). Between the low altitudes and the equatorial plane, only Polar has provided data that have been used to study electron anisotropies (Kletzing and Scudder, 1999).

Electron anisotropies in the auroral zone above the acceleration region are important from at least two different viewpoints. One viewpoint is that the return current regions adjacent to auroral arcs contain field lines where ionospheric electrons are accelerated upward, probably by downward parallel electric fields (Marklund and Karlsson, 2001). This mechanism produces upgoing electron beams. The beams we observe are often bidirectional; however to explain these using the return current region mechanism, the beams coming from the opposite hemisphere should also preserve their adiabaticity when moving through the equatorial plane, which is not self-evident.

Another viewpoint is that wave-particle interactions might also produce electron anisotropies directly (Janhunen et al., 2001, 2003a). In this case the waves are the cause of the anisotropies, and the anisotropies might, in turn, be related to other auroral processes, such as potential structure formation. This occurs because an anisotropic electron distribution, together with the mirror force, generates a parallel electric field, if the ion anisotropy differs from the electron anisotropy. This was shown by Alfvén and Fälthammar (1963) under the simplifying assumption that all particles have the same magnetic moment and energy; for a more recent and complete discussion, see, e.g. Whipple (1977). Explained in another way, an anisotropic electron distribution in the absence of parallel electric fields generates an electron density profile that varies with altitude. Unless the ion density profile has a similar altitude variation, which is very unlikely, a tendency to produce a charge imbalance results. Next, we assume that some of the electrons are cold and of ionospheric origin. If the resulting charge imbalance is greater than the cold electron density at any point, parallel potential differences of the same order of magnitude as the energy 
of the electron anisotropic component will develop, because the parallel electric field now has to be large enough to alter the motion of the magnetospheric electrons. If, on the other hand, the charge imbalance remains below the cold electron density, only small parallel fields develop, with the parallel potential being of the order of the magnitude of the cold electron temperature. Thus, the appearance or non-appearance of significant parallel potential differences depends only on the density of the anisotropic component and on the type of the anisotropy, whereas the magnitude of the potential differences, in case they appear, also depends on the temperature of the anisotropic component. In this viewpoint, electron anisotropies are expected to be correlated with auroral phenomena in general and not limited to the return current region. By looking at the appearance of the anisotropies only, anisotropies related to the return current region or to wave-particle interactions in the magnetosphere cannot necessarily be distinguished from each other.

The purpose of this paper is to gain and present eventbased and statistical knowledge of the electron anisotropies and to show that it is specifically the middle-energy anisotropies $(100-1000 \mathrm{eV})$ that probably play a role in auroral phenomena. This knowledge may be useful later in distinguishing among the two mentioned viewpoints, i.e. how important are anisotropies related to upflowing electron beams relative to those (if any) anisotropies that are produced "in situ" in the magnetosphere.

The structure of the paper is as follows. We first explain and motivate the definitions of anisotropy measures used in this paper. Thereafter we present and discuss in detail three anisotropy events observed by the HYDRA instrument on the Polar satellite. Then we present statistical results using five years of HYDRA observations and close the paper with a summary.

\section{Data processing}

To investigate the electron distribution functions in the 5000 $30000 \mathrm{~km}$ altitude range we use the Polar HYDRA data from years 1996-2000 (Scudder et al., 1995). The time resolution of the data is about $12 \mathrm{~s}$ and the energy range $2 \mathrm{eV}-28 \mathrm{keV}$. The pitch angle resolution is not fixed by the instrument because it contains 12 narrow-field sensors which are rotating with the spacecraft and each of them makes a complete energy scan in about $1.1 \mathrm{~s}$. Electrons and ions are measured by each detector on alternate energy scans. In this paper we bin the HYDRA data into $15^{\circ}$ pitch angle bins.

Usually one describes an anisotropic distribution by a Maxwellian or kappa distribution having different parallel and perpendicular temperatures. Thus, one could think that giving the temperature ratio $T_{\|} / T_{\perp}$, perhaps multiplied by the electron density, would be a good way of quantifying the magnitude of the anisotropy. However, in practice, this approach has a severe difficulty: it will turn out that almost always the electron plasma cannot be modelled by a single Maxwellian or kappa distribution, but one needs at least two such distributions (examples are given by Janhunen et al., 2001). The anisotropy usually exists in only one of these distributions. Therefore, one way of defining the anisotropy properly is to have a superposition of at least two Maxwellian or kappa distributions, where one of the populations is anisotropic. As a result, one obtains the temperature ratio of the anisotropy. This approach is perhaps useful for manual analysis of a few timesteps, but it is too complicated to be useful in a statistical study having $\sim 10^{6}$ measurements.

To get around this problem and to be able to do a statistical study, we first quantify the anisotropy simply by taking the differential energy flux $F(E, \theta)$, where $E$ is the energy and $\theta$ is the pitch angle (the dimensionality of $F$ is $\mathrm{eV} \mathrm{cm}^{-2} \mathrm{~s}^{-1} \mathrm{sr}^{-1} \mathrm{eV}^{-1}$ ) and compute the ratio $r(E)$ between the parallel and perpendicular fluxes,

$r(E)=(1 / 2)(F(E, 0)+F(E, \pi)) / F(E, \pi / 2)$.

We call $r(E)$ the relative energy-dependent anisotropy. The relative energy-dependent anisotropy is useful for colourcoded plotting as a function of time and energy, and it will be used in the example events in Sect. 3 below. Such a plot tells us at a glance in which energy range the anisotropy mainly occurs.

While the relative energy-dependent anisotropy defined above is useful in studying individual events, for statistical purposes it has three drawbacks: (1) for quantifying the anisotropy one would prefer a single number, not something which is a function of the energy, (2) being a relative number it does not tell how many electrons are actually anisotropic, (3) it is not particularly robust against instrument problems, since it depends on the differential energy flux measured in only a few pitch angle bins, two of which are exactly parallel to the magnetic field and thus not always measured reliably (unreliability exists if none of the 12 sensors points to the field-aligned direction).

The event plots in the next section will make it clear that often the anisotropy occurs mainly between $100 \mathrm{eV}$ and $1 \mathrm{keV}$ only. Motivated by this, we shall call the interval 10 $100 \mathrm{eV}$ range "low", the $100-1000 \mathrm{eV}$ range "middle" and the interval 1-10 keV range "high". Thus, to measure the anisotropy that we are mainly interested in, one should consider only the middle energy range. To overcome the first two problems in the relative energy-dependent anisotropy listed in the previous paragraph, one could, for example, average Eq. (1) over the middle-energy range and multiply by the density of middle-energy electrons. This would give a single number which is proportional to both the anisotropy and the density, i.e. the "anisotropic part" of the density (note, however, that it can also exceed the total density if $r(E)$ is large). This procedure involves two integrations and still depends on the exactly parallel pitch angle bins and thus still has the robustness problem.

The reason why we are more interested in the density than in the temperature is that the density controls the appearance or non-appearance of potential structures, while the temperature affects the magnitude of the structures, but only when 
the density is large enough that the structures appear (cf. the Introduction).

We now present the definition that we are really using. The reader can verify that it is closely related to the one just described, but it is more natural and more robust. The robustness comes about because all pitch angles are integrated over not only the exactly parallel and perpendicular ones. Let $F(E, \theta)$ denote the HYDRA electron differential energy flux as above. Then $f(E, \theta)=\left(m_{e}^{2} /\left(2 E^{2}\right)\right) F(E, \theta)$ is the distribution function. We denote the distribution function in velocity space by $\tilde{f}, \tilde{f}(v, \theta)=f(E, \theta)$, where $E=(1 / 2) m_{e} v^{2}$. Here, $\theta$ is the pitch angle as above, defined so that $\theta=0$ is parallel to $\mathbf{B}$ (downward direction in Northern Hemisphere) and $\theta=\pi$ antiparallel to $\mathbf{B}$ (upward direction in Northern Hemisphere), and $m_{e}$ is the electron mass. With this notation, we compute the anisotropic charge densities for upward and downward moving electrons from

$n_{\text {aniso }}^{\text {up }}=2 \pi \int_{\pi / 2}^{\pi} d \theta \sin \theta \int_{v_{\min }}^{v_{\max }} d v v^{2}[\tilde{f}(v, \theta)-\tilde{f}(v, \pi / 2)]$,

$n_{\text {aniso }}^{\text {down }}=2 \pi \int_{0}^{\pi / 2} d \theta \sin \theta \int_{v_{\min }}^{v_{\max }} d v v^{2}[\tilde{f}(v, \theta)-\tilde{f}(v, \pi / 2)]$

These formulas are valid as written in the Northern Hemisphere. In the Southern Hemisphere the $\theta$ integration limits are replaced by $0 . . \pi / 2$ for $n_{\text {aniso }}^{\text {up }}$ and $\pi / 2 . . \pi$ for $n_{\text {aniso }}^{\text {down }}$. The $v$ integral is restricted in a range of velocities $v_{\min }$.. $v_{\max }$ corresponding to an energy range $E_{\min } . . E_{\max }$ where $E_{\min , \max }=(1 / 2) m_{e} v_{\min , \max }^{2}$. The total anisotropic charge density is defined by

$n_{\text {aniso }}=n_{\text {aniso }}^{\text {up }}+n_{\text {aniso }}^{\text {down }}$

and the up-down difference anisotropy by

$n_{\text {aniso }}^{\text {diff }}=n_{\text {aniso }}^{\text {up }}-n_{\text {aniso }}^{\text {down }}$.

Finally, the relative anisotropy is defined by

$A_{\text {rel }}=\frac{n_{\text {aniso }}}{n_{\perp}}$,

where

$$
\begin{aligned}
n_{\perp} & =2 \pi \int_{\pi / 2}^{\pi} d \theta \sin \theta \int_{v_{\min }}^{v_{\max }} d v v^{2} \tilde{f}(v, \pi / 2) \\
& =2 \pi \int_{v_{\min }}^{v_{\max }} d v v^{2} \tilde{f}(v, \pi / 2)
\end{aligned}
$$

is the "perpendicular density".

All the $n_{\text {aniso }}$ quantities have the dimensionality of density, i.e. can be expressed in units of $\mathrm{cm}^{-3}$. Explained in words, the perpendicular distribution function is copied over all pitch angles, subtracted from the distribution function, and the result of the subtraction is integrated over the wanted velocity (energy) range to obtain the partial density corresponding to the anisotropic part of the distribution. The quantity $n_{\text {aniso }}^{\text {diff }}$ tells how much imbalance in the up/down direction there is in the anisotropy: for example, if the anisotropy consists of only upgoing electrons, $n_{\text {aniso }}^{\text {diff }}=n_{\text {aniso }}$. The relative anisotropy $A_{\text {rel }}$ is a dimensionless number indicating the significance of the anisotropy. It is positive for $T_{\|}>T_{\perp}$ type anisotropies, equal to zero for isotropic distributions and negative for $T_{\|}<T_{\perp}$ type anisotropies.

The anisotropies, as defined here, can have both positive and negative values. Negative anisotropies $\left(T_{\perp}>T_{\|}\right.$type anisotropies) turn out to be rare, at least in the middle energy range, and are not discussed in this paper.

\section{Specific events}

To obtain a more concrete picture of the electron anisotropies in the auroral zone, we now study three specific events, collected at radial distances between 3.5 and $5.5 R_{E}$, where the first one represents eveningside, the second one midnight and the third one morningside. Some kind of middle-energy anisotropies can be discerned in almost all auroral crossings. The examples selected here exhibit anisotropies that are a bit stronger and clearer than on the average, but that are not the strongest possible. The examples are from Northern Hemisphere, so a zero pitch angle means downward.

\subsection{Eveningside event - 8 May 1998}

Figure 1 shows the event that occurred on 8 May 1998, 11:35-12:35 UT. Panels $(\mathrm{a}-\mathrm{c})$ show the standard HYDRA differential energy flux panels in the downward (pitch angle range $\left.0-30^{\circ}\right)$, perpendicular $\left(75-105^{\circ}\right)$ and upward direction $\left(150-180^{\circ}\right)$ electrons, respectively. From these panels it is difficult to detect anisotropies, but they become very clear in panel (d) which is the relative energy-dependent anisotropy, (Eq. 1). The $T_{\|}>T_{\perp}$ type anisotropies that we are interested in and which are also the most common appear as red. In all HYDRA colour panels, differential energy fluxes which are smaller than $10^{5} \mathrm{eV} \mathrm{cm} \mathrm{cm}^{-2} \mathrm{~s}^{-1} \mathrm{sr}^{-1} \mathrm{eV}^{-1}$ are shown as black. In panel (d), if either the parallel or the perpendicular differential energy flux is smaller than the limit $10^{5} \mathrm{eV} \mathrm{cm}^{-2} \mathrm{~s}^{-1} \mathrm{sr}^{-1} \mathrm{eV}^{-1}$, the corresponding relative energy-dependent anisotropy is also shown as black. Additionally, points having larger than $30 \%$ statistical error (points that have less than 10 counts) are shown as black. Thus, values differing from black are guaranteed to represent values that are measured reliably. Panel (e) shows three curves: the anisotropy $n_{\text {aniso }}$ (red), the up minus down difference anisotropy $n_{\text {aniso }}^{\text {diff }}$ (green) and the relative anisotropy $A_{\text {rel }}$ (black). The scale of the red and green curves is on the left (both are in density units) and the scale of the dimensionless black curve is on the right.

In Fig. 1, Polar arrives from the polar cap and enters the auroral zone at 12:00 UT in the pre-midnight sector. Rather strong middle-energy anisotropies are detected 12:02-12:07 UT. The anisotropies also extend above $1 \mathrm{keV}$ to some extent (panel $\mathrm{d}$ ). The middle-energy anisotropy (panel e) reaches $0.2 \mathrm{~cm}^{-3}$ and the relative anisotropy $A_{\text {rel }}$ (Eq. 6) has a maximum value of 2. The anisotropies are almost up/down symmetrical because the difference anisotropy 

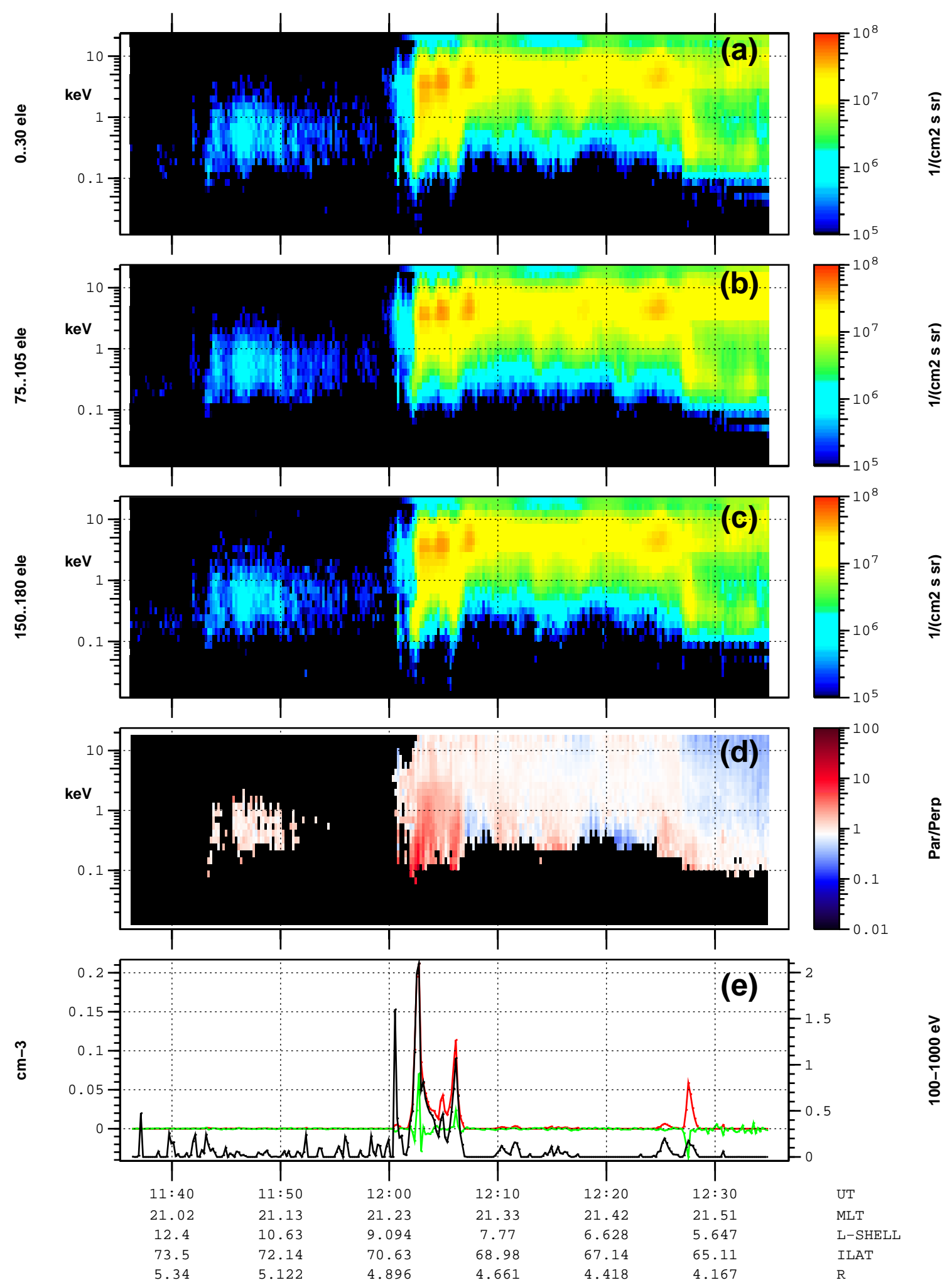

Fig. 1. HYDRA electron data for 8 May 1998, 11:40-12:35 UT. Panels from top to bottom: differential electron energy flux for (a) downgoing electrons, (b) perpendicular electrons, (c) upgoing electrons; (d) ratio of parallel to perpendicular differential energy flux (energy-dependent relative anisotropy $r(E)$, Eq. (1)); and (e) three middle-energy anisotropy curves: $n_{\text {aniso }}$ (red) and $n_{\text {aniso }}^{\text {diff }}$ (green) with scale on the left, and relative anisotropy $A_{\text {rel }}$ (black, with scale on the right). 
ELE 19980508, 12:02:12 .. 12:02:24

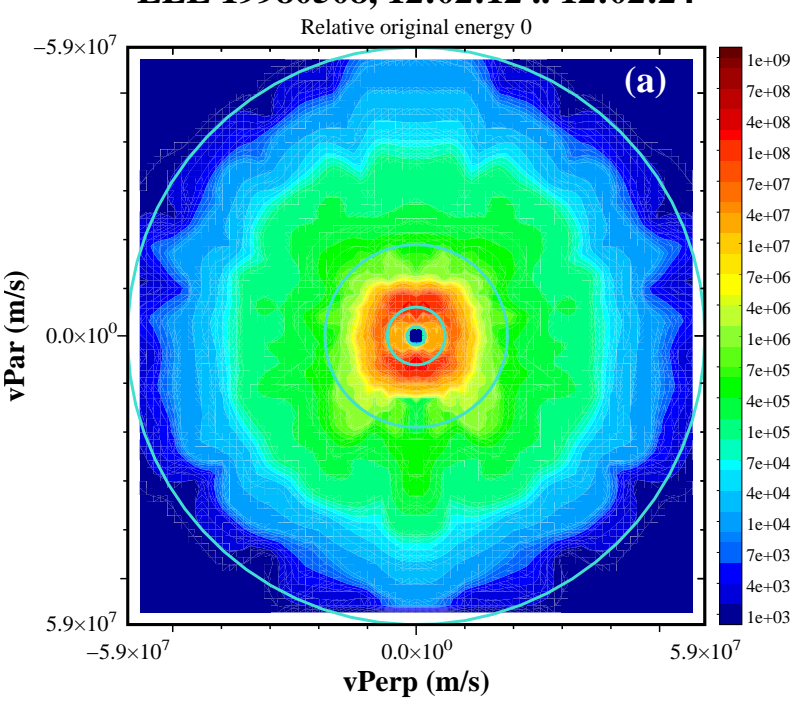

ELE 19980508, 12:02:36 .. 12:02:48

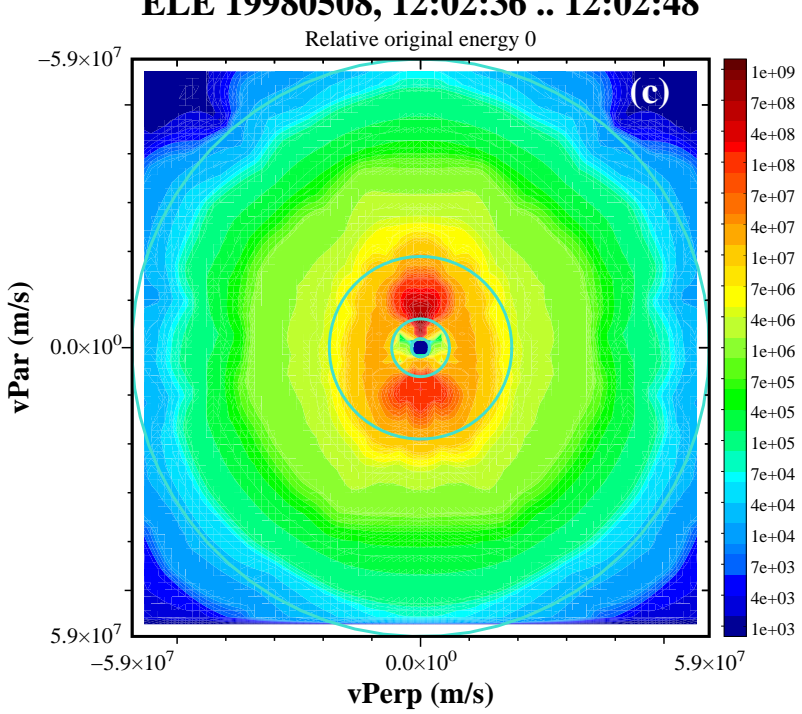

ELE 19980508, 12:02:24 .. 12:02:36

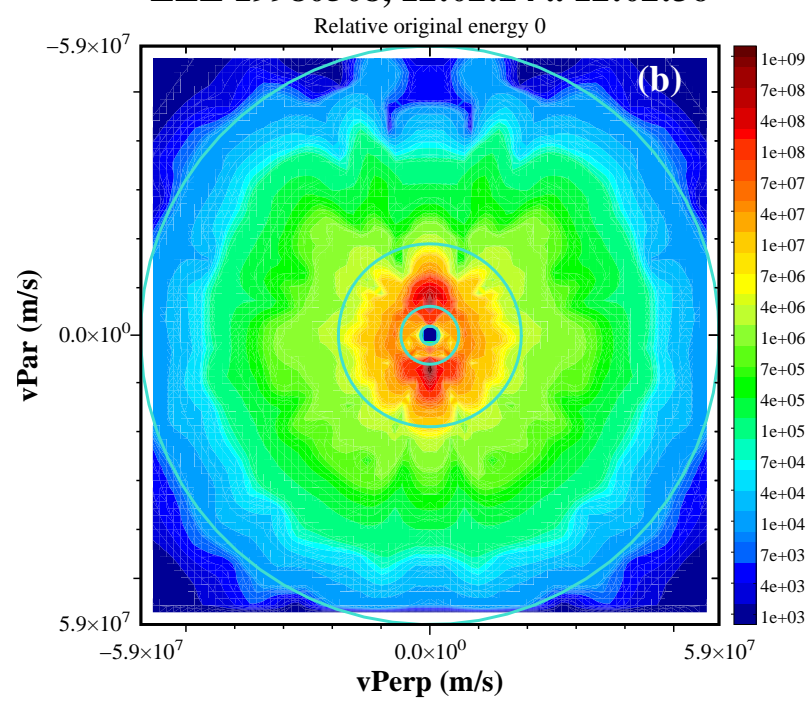

ELE 19980508, 12:02:48 .. 12:03:0

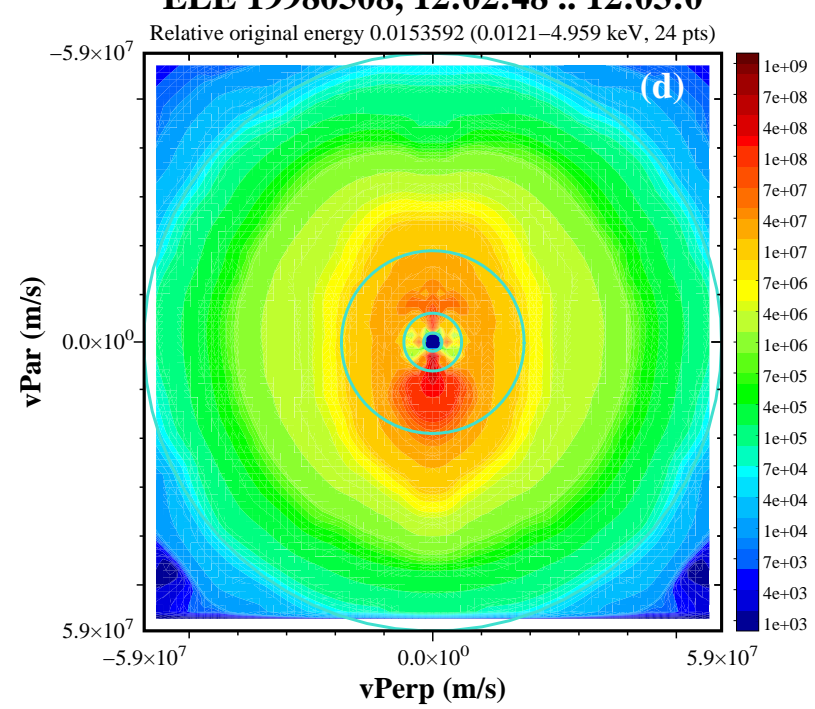

Fig. 2. Electron distribution function $\left(\mathrm{cm}^{-2} \mathrm{~s}^{-1} \mathrm{sr}^{-1} \mathrm{keV}^{-2}\right.$ ) for four successive 12-s intervals on 8 May 1998 event, starting at (a) 12:02:18 UT, (b) 12:02:30, (c) 12:02:42 UT, (d) 12:02:54. Velocities corresponding to $10 \mathrm{eV}, 100 \mathrm{eV}, 1 \mathrm{keV}$ and $10 \mathrm{keV}$ energies are shown by blue circles; notice that the innermost circle is very small. Magnetic field is oriented downward, parallel to the positive vertical axis, and positive parallel velocity is along the magnetic field, i.e. downward, and the loss cone, if any, appears on the top of each panel.

(green curve in panel e) is smaller than the anisotropy itself, except for a brief moment near 12:27 UT. This example was chosen mainly to demonstrate that occasionally the middleenergy anisotropies can extend to higher than $1 \mathrm{keV}$ energies.

Figure 2 shows the electron distribution function at four successive intervals, separated by $12 \mathrm{~s}$, around the strongest anisotropy peak at 12:02-12:03 UT. The four circles where the innermost one is barely visible correspond to 10,100 , 1000 and $10000 \mathrm{eV}$ energies. The first interval (panel a) displays almost no anisotropy, whereas the second one (panel b) exhibits a clear middle-energy anisotropy. This anisotropy is slightly atypical in that there are relatively few electrons below $100 \mathrm{eV}$ energy. The 3rd interval (panel c) also displays a clear anisotropy, and in the 4th interval (panel d) there still exists anisotropy, with a modest predominantly downward character. Now the anisotropy also extends to higher energies above $1 \mathrm{keV}$. In all panels an almost isotropic high-energy Maxwellian electron distribution is seen which is likely to be of magnetospheric origin. This is a feature which is nearly invariably associated with the middle-energy anisotropies. 

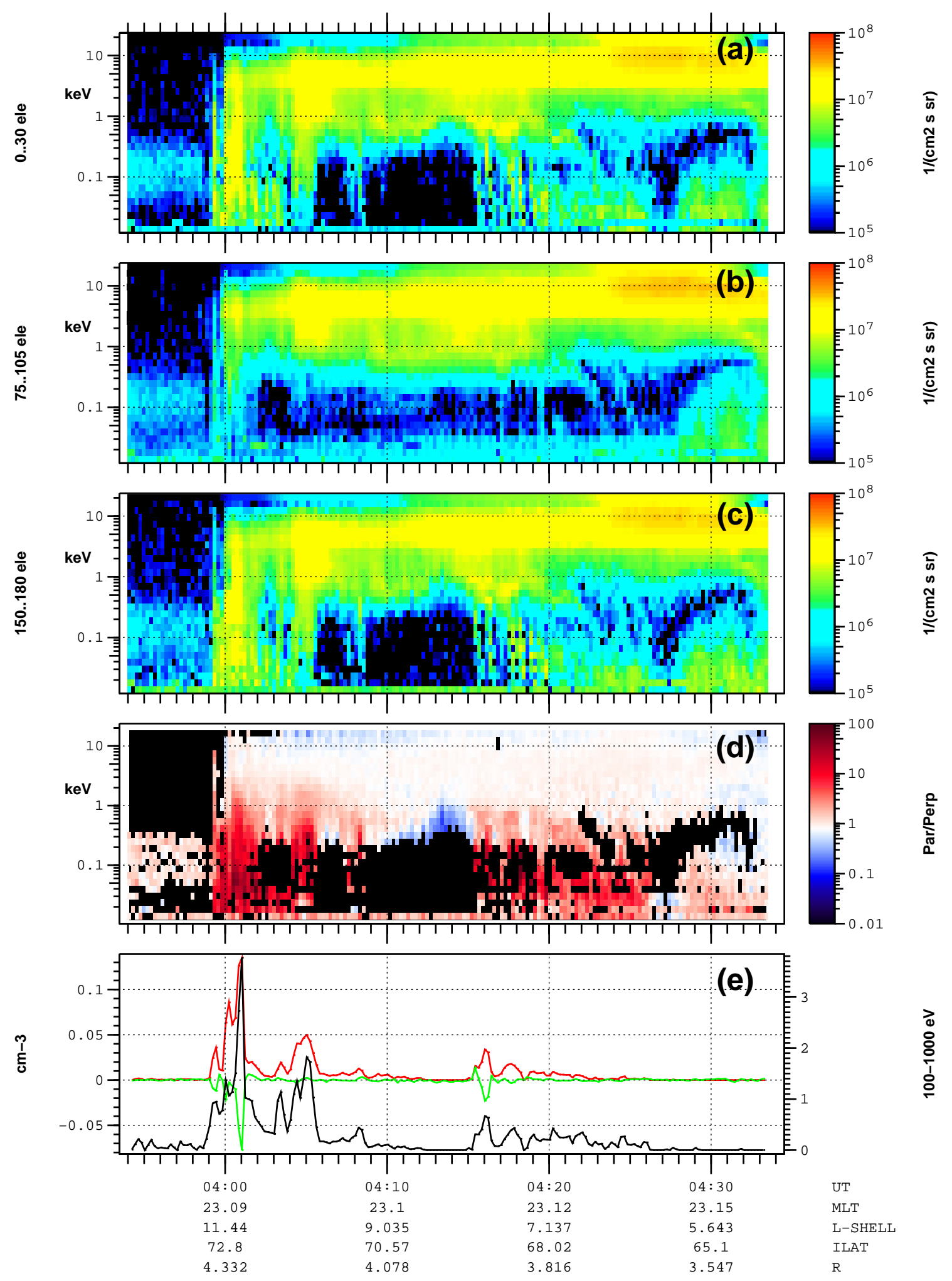

Fig. 3. Same as Fig. 1 but for 22 April 1998, 03:55-04:35 UT. 

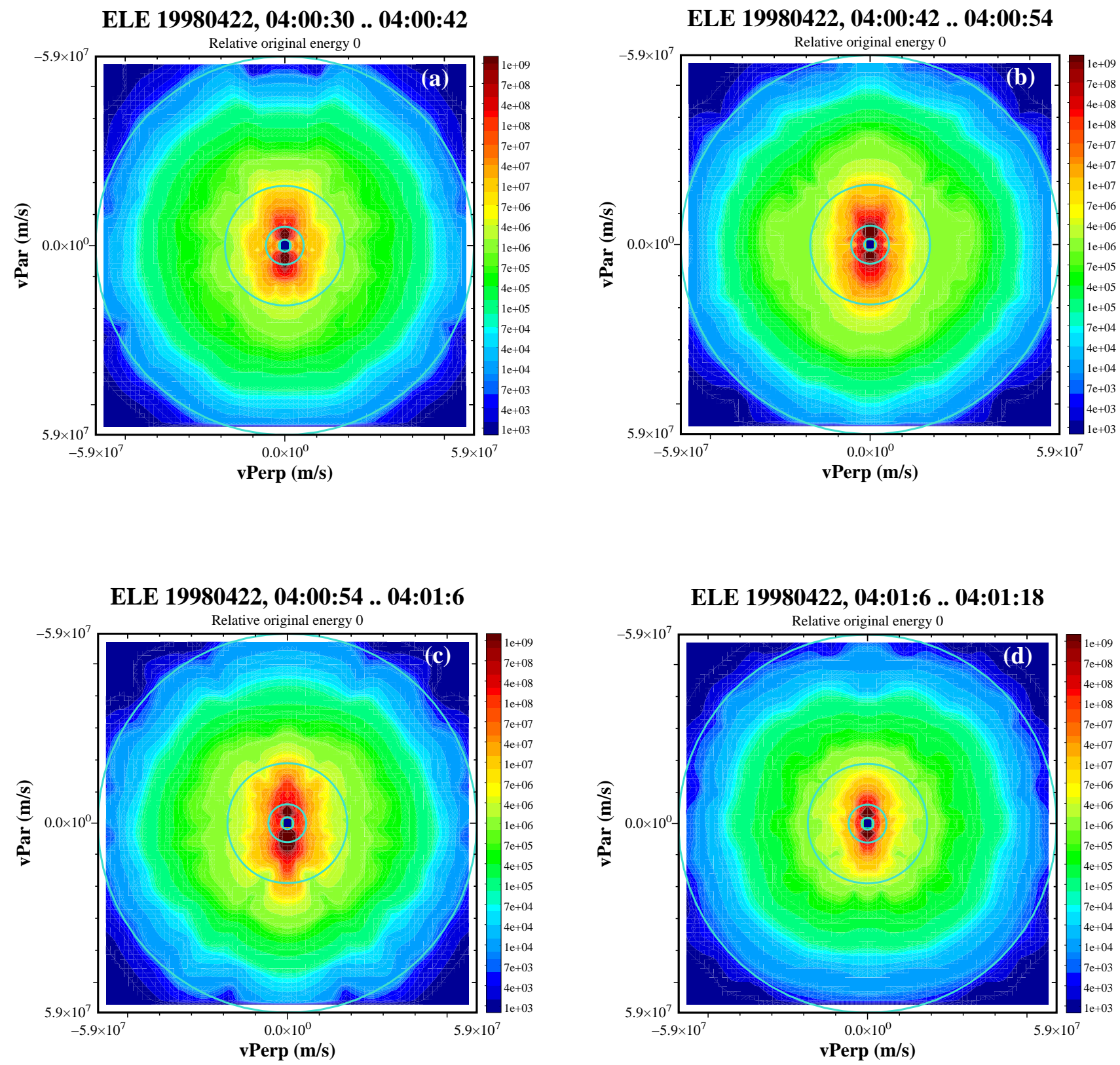

Fig. 4. Electron distribution functions $\left(\mathrm{cm}^{-2} \mathrm{~s}^{-1} \mathrm{sr}^{1} \mathrm{keV}^{-2}\right.$ ) for four successive 12-s intervals on 22 April 1998: (a) 04:00:36 UT, (b) 04:00:48, (c) 04:01:00, (d) 04:01:12. Same format as in Fig. 2.

The high-energy isotropic electrons have not experienced any parallel acceleration.

\subsection{Midnight event - 22 April 1998}

Figure 3 shows the event that occurred on 22 April 1998, 03:55-04:35 UT, near the local midnight. The format of the figure is similar to Fig. 1. Again, Polar moves from the polar cap into the auroral zone at 03:58 UT. Strong low and middleenergy anisotropies can be detected over the whole auroral interval. Highest anisotropies occur 03:59-04:01 UT (red line in panel e), when the relative anisotropy $A_{\text {rel }}$ (black line in panel e) reaches almost 4. The strongest peak at 04:01 UT is predominantly downward (negative green curve in panel e), but otherwise the anisotropies are more or less up/down symmetrical.

Figure 4 presents four distribution functions, taken at four successive intervals separated by $12 \mathrm{~s}$ around the strongest anisotropy peak 04:01 UT. They show that in this case the anisotropies did not vary as rapidly as in the 8 May 1998 example. All four intervals show roughly similar and rather typical (strong) middle-energy anisotropies.

In this event there are also low-energy up- and downgoing electron beams visible in Fig. 3, for example, around 

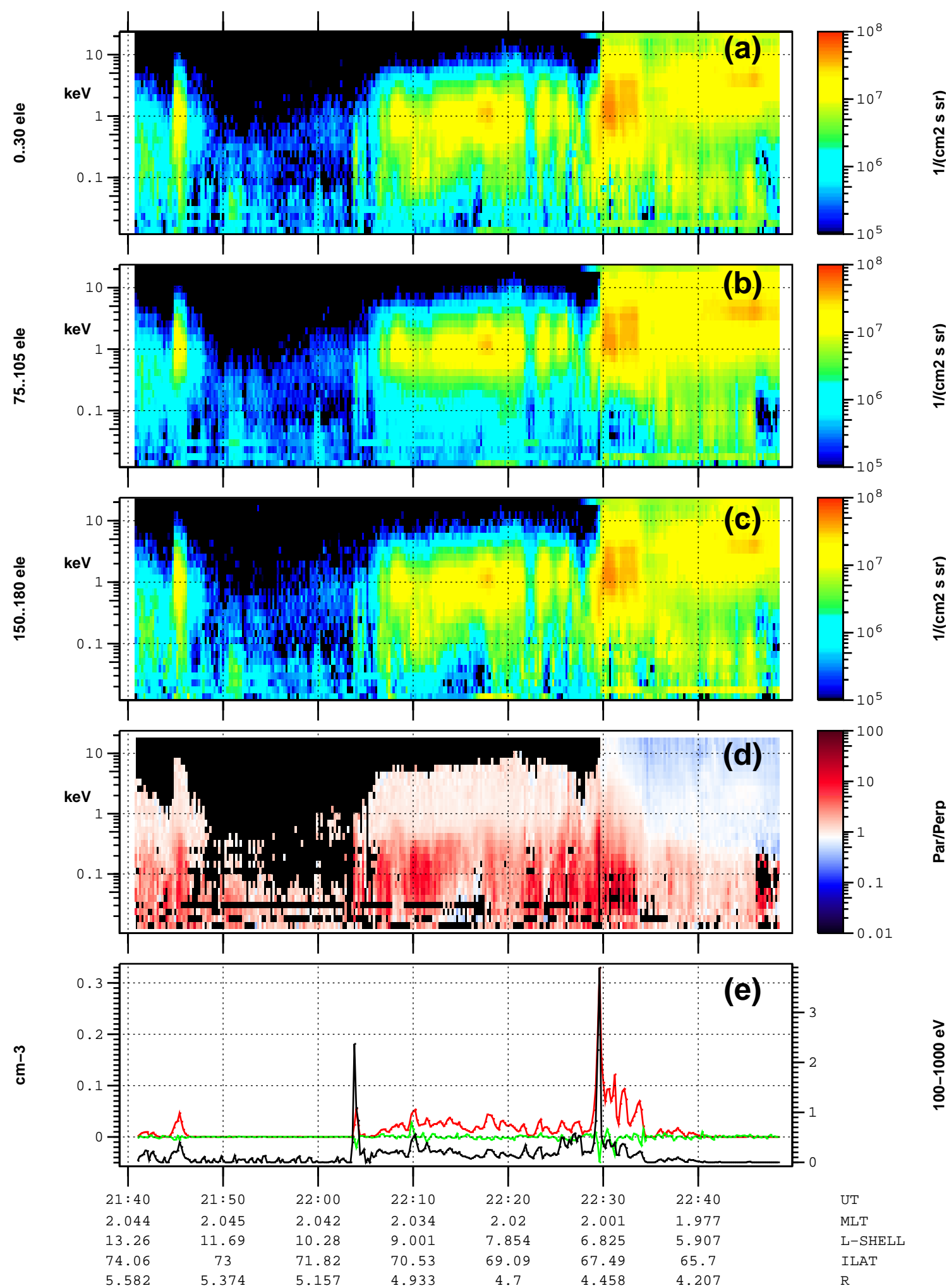

Fig. 5. Same as Fig. 1 but for 10 March 1998, 21:40-22:50 UT. 
ELE 19980310, 22:29:18 .. 22:29:30

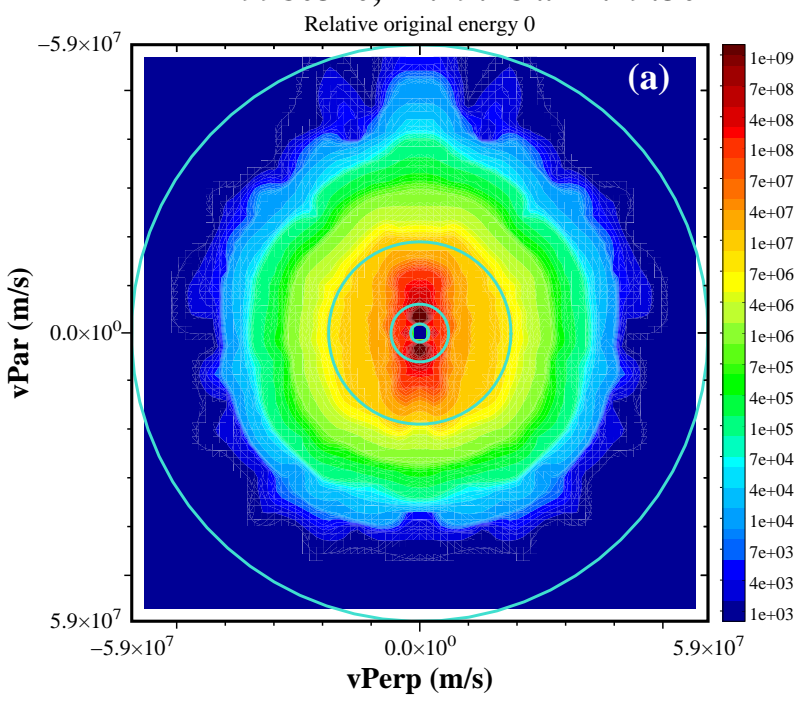

ELE 19980310, 22:29:42 .. 22:29:54

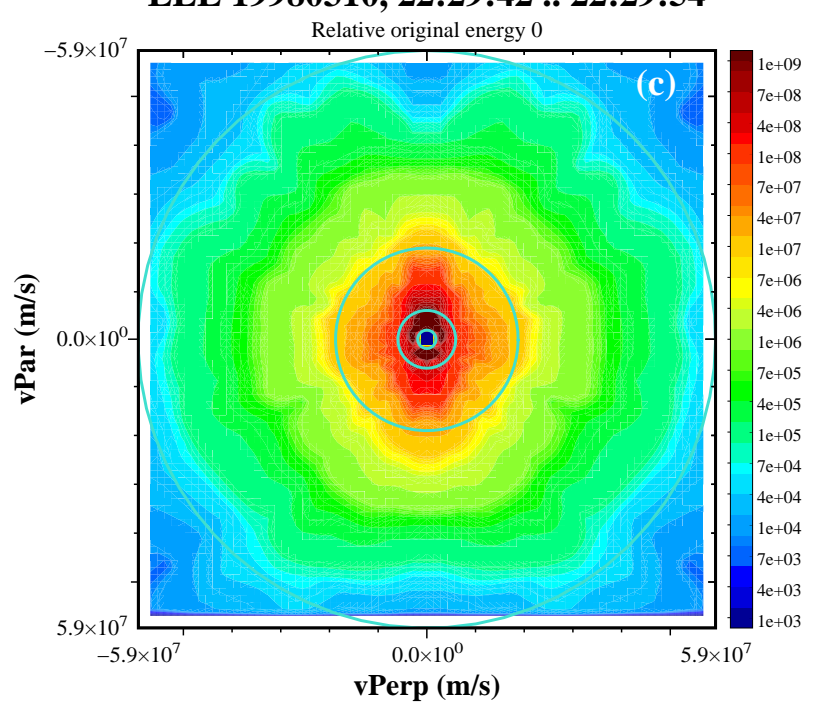

ELE 19980310, 22:29:30 „22:29:42

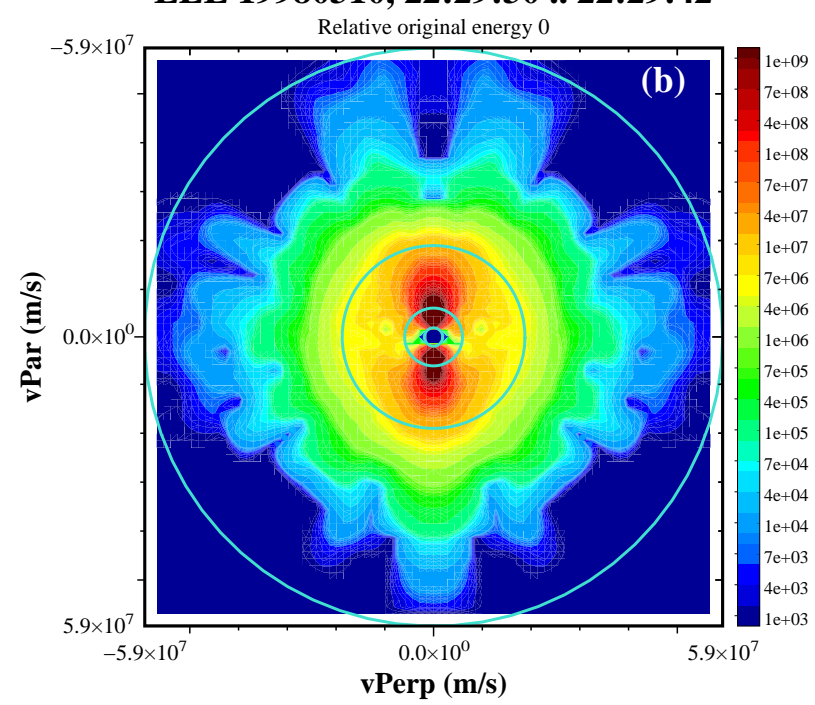

ELE 19980310, 22:29:54 .. 22:30:6

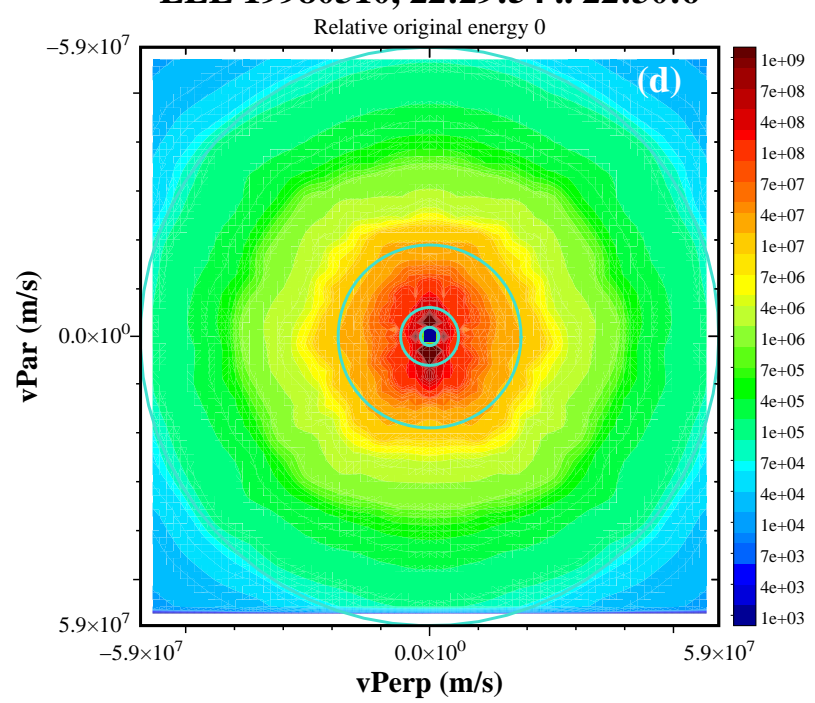

Fig. 6. Electron distribution functions $\left(\mathrm{cm}^{-2} \mathrm{~s}^{-1} \mathrm{sr}^{1} \mathrm{keV}^{-2}\right.$ ) for four successive 12-s intervals on 10 March 1998: (a) 22:29:18 UT, (b) 22:29:36, (c) 22:29:48, (d) 22:30:00. Same format as in Fig. 2.

04:18 UT. Since this activity is below $100 \mathrm{eV}$, it does not contribute to panel (e).

\subsection{Morningside event - 10 March 1998}

Figure 5 shows an event that occurred on 10 March 1998, 21:40-22:45 UT, in the post-midnight sector. There is anisotropy all the way from 22:05 to 22:35 UT. The strongest peak appears close to 22:30 UT. The anisotropies during this event are at somewhat lower energies than in the other two examples, now extending all the time, also well below $100 \mathrm{eV}$, and there are hardly any anisotropies above $1 \mathrm{keV}$, even though strong electron fluxes exist around $1 \mathrm{keV}$ (see the top three panels).

The distribution functions taken around 22:30 UT (Fig. 6) show how the anisotropy grows and reaches its peak and then suddenly becomes reduced. At the same time the temperature of the high-energy electron population increases (or a new population appears). When the anisotropy reaches its peak (panel b) the whole distribution is irregular, which suggests that rapid temporal variations are going on during this 12-s integration. 


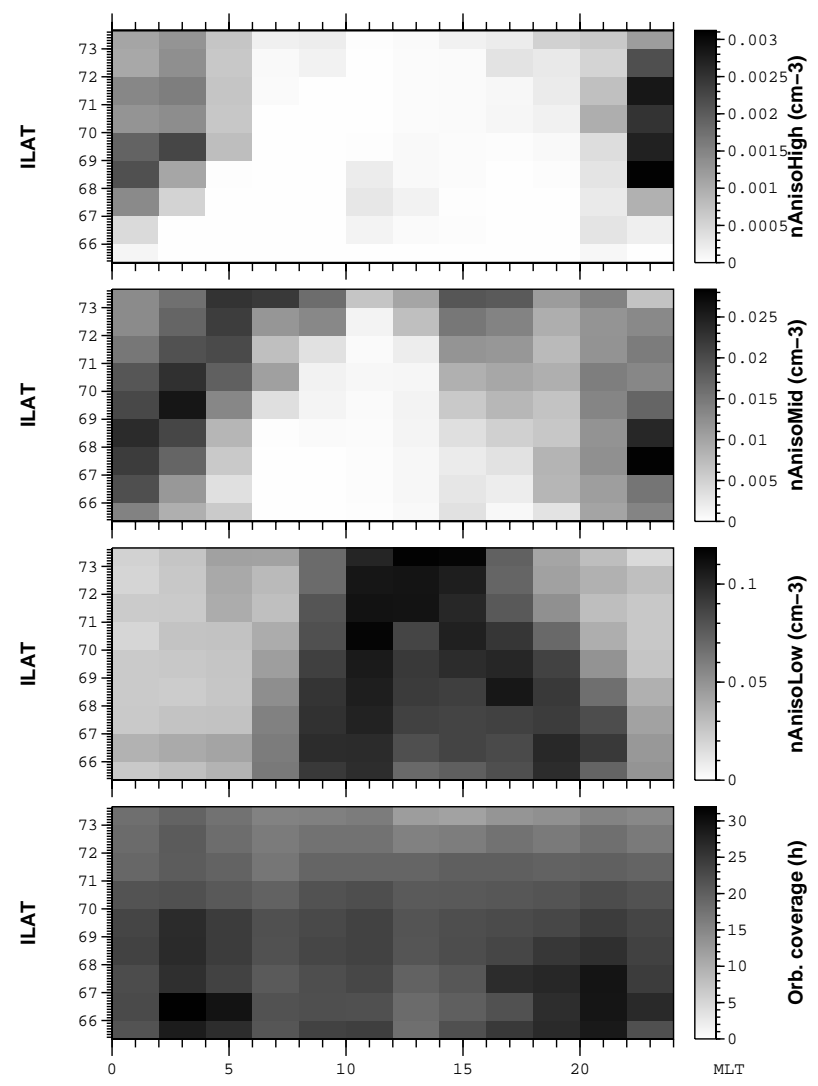

Fig. 7. 90th percentiles of electron anisotropies in different energy ranges as a function of MLT and ILAT and integrated over radial distances $2.5--6 R_{E}$, for high-energy (1-10 keV; top panel), middle-energy (100-1000 eV; second panel), low-energy (10-100 eV; third panel). The bottom panel shows the orbital coverage in hours. The 90th percentile is defined as the value which is such that $90 \%$ of the measured values are smaller and $10 \%$ are larger.

In all three events presented, strong anisotropy peaks accompany the polar cap boundary, which is sharply defined. Among auroral crossings of Polar, this kind of behaviour is relatively common, although by no means a rule. Also, in the events shown, some of the anisotropy peaks occur together with sudden changes in the high energy electron distribution. This behaviour is not very typical, but occurs only in some events.

\section{Statistical results}

\subsection{MLT and ILAT}

Figure 7 shows the distribution of the 90th percentile of the electron anisotropies for three energy ranges, as a function of MLT and ILAT. The bottom panel shows the number of hours measured by the instrument in each bin. The radial distance range included in the plot is $2.5-6 R_{E}$. The 90th percentile is defined as the value which is such that $90 \%$ of the measured values are smaller and $10 \%$ are larger. The motivation for selecting the 90 th percentile is that $10 \%$ is the typical oc-
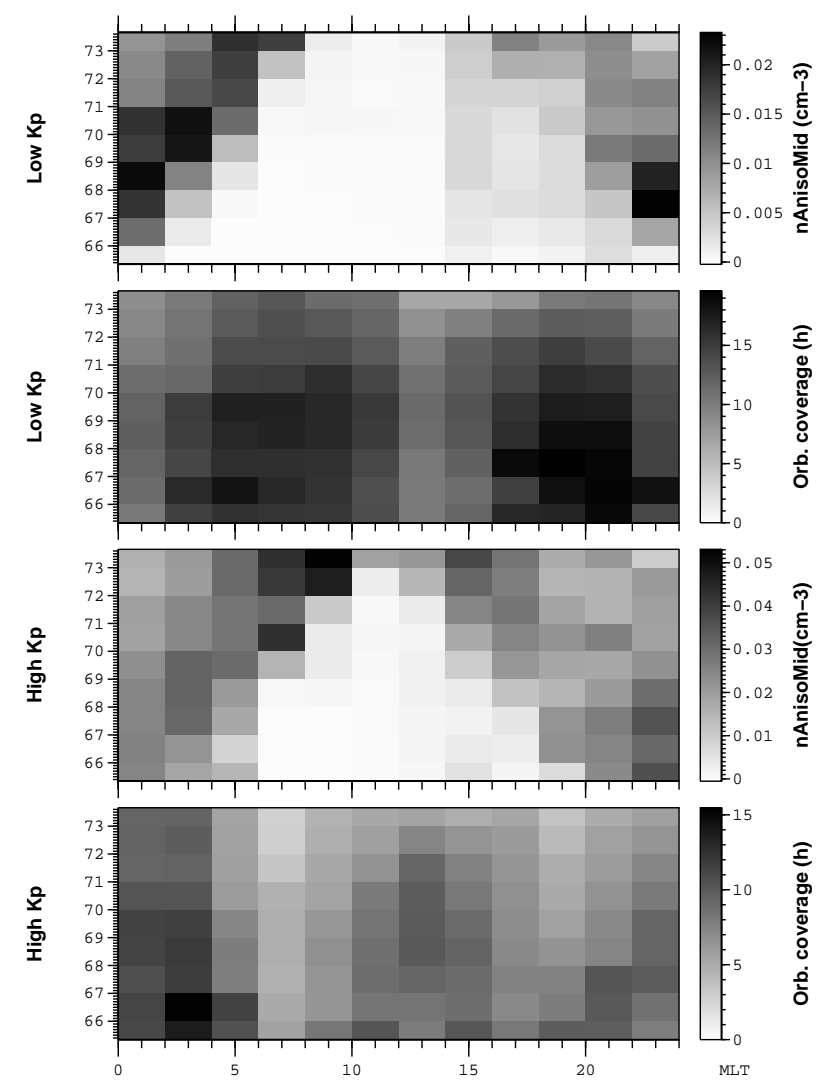

Fig. 8. Middle-energy (100-1000 eV) anisotropy dependence on $K_{p}$ index. First panel: 90th percentile of middle-energy (100$1000 \mathrm{eV})$ anisotropies for low $K_{p}\left(K_{p} \leq 2\right)$, second panel: orbital coverage for low $K_{p}$, third panel: 90 th percentile for high $K_{p}$ $\left(K_{p}>2\right)$, fourth panel: orbital coverage for high $K_{p}$. The vertical axis in each panel is the invariant latitude (ILAT).

currence frequency of auroral arc-related phenomena within the auroral zone. If one uses the 75th percentile (i.e. the upper quartile) instead of the 90th percentile, for example, the values in all panels become smaller but the patterns remain qualitatively very similar. The high-energy range (top panel) has the lowest values for the anisotropy, and these anisotropies occur mainly near the midnight auroral zone. The middle-energy anisotropies (second panel from the top) follow the auroral oval pattern, with a clear MLT dependence, with anisotropies being more common around midnight than elsewhere. The third panel from top shows the low-energy anisotropies, which are seen to be mainly a dayside phenomenon, being more common in the pre-noon sector than in the post-noon sector. Although the dayside is not the primary emphasis of this paper we remark that the preference of the low-energy anisotropies to appear before noon might be due to $\mathbf{E} \times \mathbf{B}$-drifting cold plasma flowing away from the Earth in the post-noon sector which follows from the combined effect of magnetospheric convection and corotation (Kivelson and Russell (1995), Fig. 10.25 on p. 316).

The high-energy anisotropies could possibly give rise to potential structures of large magnitude, if the density imbal- 
$[\mathrm{t}]$
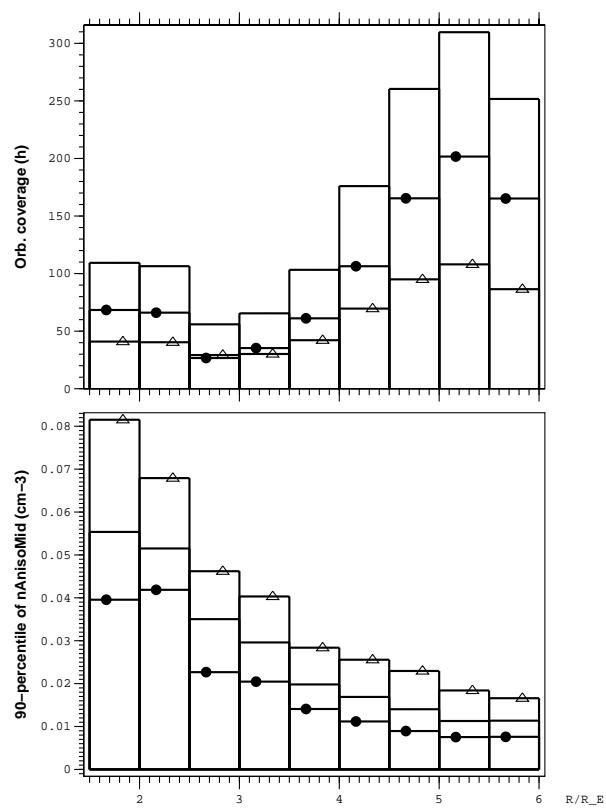

Fig. 9. Bottom panel: Radial dependence of the 90th percentile of middle-energy $\left(100-1000 \mathrm{eV}\right.$ ) anisotropies for small $K_{p}$ (solid line with filled circles, $K_{p} \leq 2$ ) and large $K_{p}$ (solid line with triangles, $K_{p}>2$ ) measured at 65:74 ILAT and 18:06 MLT. Solid line without symbols shows results for low and high $K_{p}$ combined together. Top panel: Orbital coverage in hours in each radial bin.

ance produced by them exceeds the colder component densities at some altitude (at low enough altitude one can always find cold plasma, so the question is not whether cold plasma exists but at what altitude it exists). However, the anisotropic densities are so small that this is not likely to happen. Thus, the high energy anisotropies are probably not very significant as far as auroral potential structures are concerned.

The low-energy range $(10-100 \mathrm{eV})$ probably cannot be trusted completely, since it is affected, for example, by the spacecraft potential. It is possoble that the anisotropies that we call "middle-energy" actually rather often extend below $100 \mathrm{eV}$, although the instrument cannot always reliably detect them.

If one produces a plot similar to Fig. 7 separately for upgoing and downgoing anisotropies $n_{\text {aniso }}^{\text {up }}$ and $n_{\text {aniso }}^{\text {down }}$, the plots are nearly identical, i.e. there is no clear difference in the statistical properties of upgoing and downgoing anisotropies.

\section{$4.2 K_{p}$}

Figure 8 shows how the middle-energy (100-1000 eV) anisotropy 90 th percentile depends on the $K_{p}$ index. We see that when $K_{p}$ increases, the middle-energy anisotropies move towards lower latitudes. This is a further confirmation that the middle-energy anisotropies are associated with auroral processes, since the auroral oval also resides at lower latitudes during high $K_{p}$ (Feldstein and Starkov, 1967). The aniso- tropy magnitude generally increases with an increasing $K_{p}$ index.

\subsection{Other effects}

Without showing plots, we mention a few results for the anisotropies. First, the middle-energy anisotropies are almost symmetric in the up/down direction, which means that most of the electrons do not precipitate into the atmosphere or originate from it. Second, there is no clear solar illumination dependence, saying that it is not important to the anisotropy results whether or not the ionospheric footpoint is sunlit.

\subsection{Altitude dependence}

The bottom panel of Fig. 9 shows the 90th percentile of the middle-energy anisotropies against the geocentric radial distance $R$ for small ( $\leq 2)$ and large $(>2) K_{p}$ values. The top panel presents the number of hours the satellite spent in each $0.5 R_{E}$ radial distance bin. We notice that for increasing $K_{p}$ the anisotropy magnitude increases. Furthermore, the anisotropy magnitude increases with decreasing altitude, which is natural because the stronger the anisotropy (the larger the ratio $T_{\|} / T_{\perp}$ ), the more the density follows the flux tube scaling (Eq. (7) and Fig. 1 of Janhunen and Olsson, 2002a). It could also partly be caused by failing to detect very field-aligned anisotropies at high altitudes, because one of the detectors is not always looking exactly at field-aligned direction. Notice also that the high-altitude anisotropies should be more field-aligned than the low-altitude ones because of the mirror force.

\section{Summary and discussion}

In this paper we have investigated middle-energy electron anisotropies in the auroral zone, using observations of electron distribution functions by the HYDRA instrument from the Polar satellite and using a novel definition of anisotropy which is capable of quantifying anisotropies occurring in a limited energy range. The major findings are as follows.:

1. Most often the $T_{\|}>T_{\perp}$ type anisotropies are limited to a certain energy range, typically $\sim 100-1000 \mathrm{eV}$.

2. Almost always there is simultaneously an isotropic electron distribution at higher energies.

3. Often the anisotropies are up/down symmetric, although cases with net upward or downward electron flow also occur.

4. The MLT-ILAT distribution of middle-energy anisotropies (100-1000 eV) obeys that of the average auroral oval (Fig. 7). The distributions of the low and highenergy anisotropies are more irregular. This suggests that it is specifically the middle-energy anisotropies that have something to do with auroral acceleration processes. 
5. When the $K_{p}$ index increases, the middle-energy anisotropies appear at lower ILAT in all MLT sectors, as is expected for an auroral oval related process. Their 90th percentile also increases with increasing $K_{p}$.

6. The altitude dependence of the anisotropies is smooth as one would expect, because the electrons have high mobility along the magnetic field and thus the anisotropies spread rapidly to different altitudes.

7. Within the auroral zone and at about $4 R_{E}$ radial distance, the 90th percentile of the middle-energy (100$1000 \mathrm{eV}$ ) anisotropic density is $\sim 0.02-0.03 \mathrm{~cm}^{-3}$. The anisotropic density decreases with increasing radial distance $R$, so that it is roughly proportional to $R^{-1.8}$, which can be deduced from Fig. 9.

In order to assess the role of the middle-energy anisotropies further using observational studies, one should find their statistical correlation with other phenomena occurring on auroral field lines, such as broad-band wave activity. If the waves are producing the anisotropies, then there should exist a correlation between them, as is recently found in an event study by Janhunen et al. (2001).

A possible mechanism for producing middle-energy electron anisotropies is to have waves whose parallel phase velocity is in Landau resonance with the thermal speed of electrons. Recent particle simulations have shown that ion Bernstein waves driven to be unstable by a hot ion shell distribution can energise $\sim 100 \mathrm{eV}$ electrons at a rate of $80 \mathrm{eV} / \mathrm{s}$ (Janhunen et al., 2003a). Since the travel time of a $100 \mathrm{eV}$ electron (parallel energy) through the altitude range of, say, $4 R_{E}$ is $4 \mathrm{~s}$, such electrons may gain several hundred $\mathrm{eV}$ of extra parallel energy during one trip through the region. Because of the mirror force and convergent flux tube geometry, parallel electron energisation should also produce macroscopic charge separation effects. It has been demonstrated using a special type of electrostatic hybrid simulations that parallel electron energisation may lead to self-consistent auroral potential structure formation (Janhunen and Olsson, 2002a).

If one tries to build a synthesis of the new results mentioned in this paragraph, the following picture tends to emerge: (1) Ion Bernstein or lower hybrid waves are driven unstable by some free energy source, possibly a hot ion shell distribution (Janhunen et al., 2003a). One way to produce ion shell distributions is by time of flight effects of ions injected from the reconnection X line. (2) Middle-energy electrons (the present paper) are energised by the waves in the parallel direction with a Landau resonance mechanism (Janhunen et al., 2001, 2003a). (3) The parallel energisation of electrons leads to charge separation effects and auroral potential structure formation taking place below $\sim 4 R_{E}$ radial distance (Janhunen et al., 1999; Janhunen and Olsson, 2002a). (4) The presence of the potential structure gives the characteristic inverted- $\mathrm{V}$ shape to low-altitude electron distributions (Janhunen and Olsson, 2000), generates upgoing ion beams (Janhunen et al., 2003b) and a density cavity (Janhunen et al., 2002b). Alfvénic wave acceleration probably modifies this picture in dynamic events such as substorm onsets. The middle-energy electron anisotropies are thus one important link in a relatively complicated chain of energy flow from the reconnection $\mathrm{X}$-line to inverted-V electron precipitation.

Acknowledgements. We are grateful to Craig Kletzing for providing the HYDRA data and for giving many useful comments. The work of PJ was supported by the Academy of Finland and that of $\mathrm{AO}$ and AV by the Swedish Research Council.

The Editor in Chief thanks V. Sergeev and another referee for their help in evaluation this paper.

\section{References}

Alfvén, H. and Fälthammar, C. G.: Cosmical Electrodynamics, Clarendon, Oxford, 1963.

Collin, H. L., Sharp, R. D., and Shelley, E. G.: The occurrence and characteristics of electron beams over the polar regions, J. Geophys. Res., 87, 7504-7511, 1982.

Feldstein, Y. I. and Starkov, G. V.: Dynamics of auroral belt and polar geomagnetic disturbances, Planet. Space Sci., 15, 209-229, 1967.

Hada, T., Nishida, A., Terasawa, T., and Hones, E .W., Jr.: Bidirectional electron pitch angle anisotropy in the plasma sheet, J. Geophys. Res., 86, $11211-11$ 224, 1981.

Janhunen, P., Olsson, A., Mozer, F. S., and Laakso, H.: How does the U-shaped potential close above the acceleration region? A study using Polar data, Ann. Geophysicae, 17, 1276-1283, 1999.

Janhunen, P., Olsson, A. W., Peterson, K., Laakso, H., Pickett, J. S., Pulkkinen, T. I., and Russell, C. T.: A study of inverted-V auroral acceleration mechanisms using Polar/Fast Auroral Snapshot conjunctions, J. Geophys. Res., 106, 18 995-19 011, 2001.

Janhunen, P. and Olsson, A.: New model for auroral acceleration: O-shaped potential structure cooperating with waves, Ann. Geophysicae, 18, 596-607, 2000.

Janhunen, P. and Olsson, A.: A hybrid simulation model for a stable auroral arc, Ann. Geophysicae, 20, 1603-1616, 2002a.

Janhunen, P., Olsson, A., and Laakso, H.: Altitude dependence of plasma density in the auroral zone, Ann. Geophysicae, 20, 17431750, 2002b.

Janhunen, P., Olsson, A., Vaivads, A., and Peterson, W. K.: Generation of Bernstein waves by ion shell distributions in the auroral region, Ann. Geophysicae, in press, 2003a.

Janhunen, P., Olsson, A., and Peterson, W. K.: The occurrence frequency of upward ion beams in the auroral zone as a function of altitude using Polar/TIMAS and DE-1/EICS data, Ann. Geophysicae, in press, 2003b.

Kivelson, M. and Russell, C. T.: Introduction to space physics, Cambridge University Press, 1995.

Kletzing, C. A. and Scudder, J. D.: Auroral-plasma sheet electron anisotropy, Geophys. Res. Lett., 26, 971-974, 1999.

Klumpar, D. M., Quinn, J. M., and Shelley, E. G.: Counterstreaming electrons at the geomagnetic equator near $9 R_{E}$, J. Geophys. Res., 93, 1295-1298, 1988.

Lin, C. S., Mauk, B., Parks,G. K., DeForest, S., and McIlwain, C. E.: Temperature characteristics of electron beams and ambient particles, J. Geophys. Res., 84, 2651-2654, 1979.

Marklund, G. T. and Karlsson, T.: Characteristics of the auroral particle acceleration in the upward and downward current regions, Phys. Chem. Earth, 26, 81-96, 2001. 
Richardson, J. D., Fennell, J. F. and Croley, Jr., D. R. : Observations of field-aligned ion and electron beams from SCATHA (P78-2), J. Geophys. Res., 86, 10 105-10 110, 1981.

Scudder, J. D., Hunsacker, F., Miller, G., Lobell, J., Zawistowski, T., Ogilvie, K., Keller, J., Chornay, D., Herrero, F., Fitzenreiter, R., and 12 coauthors: Hydra - A 3-dimensional electron and ion hot plasma instrument for the Polar spacecraft of the GGS mission, Space Sci. Rev., 71, 459-495, 1995.
Sergeev, V. A., Baumjohann, W., and Shiokawa, K.: Geophys. Res. Lett., 28, 3813-3816, 2001.

Sharp, R. D., Shelley, E. G., Johnson, R. G., and Ghielmetti, A. G.: Counterstreaming electron beams at altitudes of $\sim 1 R_{E}$ over the auroral zone, J. Geophys. Res., 85, 92-100, 1980.

Whipple, E.C., Jr.: The signature of parallel electric fields in a collisionless plasma, J. Geophys. Res., 82, 1525-1531, 1977. 